\title{
The Diagnostic role of Soluble (sAxl) Level in Patients with Hepatocellular Carcinoma compared to Alpha-fetoprotein
}

Ayman M. El Lehleh (MD) ${ }^{1}$, Nasser M. Abd Elbary (MD) ${ }^{2}$, Reham Elzayat $(\mathrm{MD})^{\mathbf{3}}$, Ahmed R. El-Gazzarah (MD) ${ }^{\mathbf{1}}$, Naglaa S. Elabd (MD) ${ }^{\mathbf{1}}$

${ }^{1}$ Department of Tropical Medicine, Faculty of Medicine, Menoufia University, Menoufia, Egypt.

${ }^{2}$ Department of Clinical Oncology, Faculty of Medicine, Menoufia University, Menoufia, Egypt.

${ }^{3}$ Department of Clinical pathology, Faculty of Medicine, Menoufia University, Menoufia, Egypt.

Corresponding Author Naglaa S. Elabd, M.D

Mobile:

00201092304322

E mail: naglaa_elabd@yahoo. com

Key words: HCC, cirrhosis, Alphafetoprotein, sAxl
Background and Aim: Hepatocellular carcinoma deemed for the plurality of primary liver cancers. Malignancy of the liver is the fourth most popular etiology of cancer mortality worldwide. The high mortality of HCC cases is linked to the delay in diagnosis. Aberrant expression of Axl frequently occurs in many malignancies and is critical for promoting cell proliferation, migration, angiogenesis and metastasis. Axl deregulated activation or expression is linked to resistance to targeted cancer therapies. we aimed to to clear up the diagnostic role of sAXL in HCC patients.

Methods: Study included 90 participants; 40 HCC patients on top of liver cirrhosis, 30 patients with liver cirrhosis and twenty healthy subjects (controls). CBC, liver and kidney function tests, alphafetoprotein, abdominal ultrasound and triphasic CT were performed. sAxl was assessed by ELISA.
Results: sAxl was considerably higher in HCC patients than in cirrhotic and control participants $(\mathrm{p}<0.001)$, with higher levels in cirrhotic patients than controls $(\mathrm{p}<0.001)$. sAxl can differentiate early HCC cases from patients with cirrhosis $(\mathrm{p}<0.001)$. ROC curve analysis showed that sAxl has sensitivity $92.5 \%$, specificity $93.3 \%$ and AUC 0.949 at cut off value $>63.5$, AFP at cut off $>40.34$ has sensitivity, specificity and AUC 57.5\%, $60 \%$ and 0.675 respectively. The combination of AFP and sAxl at related cut off points has higher sensitivity $(97.5 \%)$ and AUC 0.972 in predicting HCC in cirrhotic patients. sAxl was positively correlated with tumor size.

Conclusion: sAxl outperforms AFP in detecting $\mathrm{HCC}$, especially in early $\mathrm{HCC}$ and in AFP-negative HCC. Combination of sAxl with AFP improved the sensitivity for early HCC diagnosis.

\section{INTRODUCTION}

Hepatocellular carcinoma shows the cause for the majority of primary liver cancers. Globally, malignancies of the liver represent the fourth most common etiology of cancer mortality as well as they assorted as the sixth in terms of incident cases. Based on the annual projections, the World Health Organization (WHO) speculates that more than 1 million patients will die in 2030 from liver cancer [1].

Worldwide, the preponderance HCC cases are diagnosed at late stages, leading to a median survival of about one to 4 years, accounting on the country [2]. Whilst, HCC cases that are diagnosed at an early stage of disease development exhibit a promising five-year survival rate of seventy percent following curative treatment strategies as liver transplantation or tumor resection [3].

Patients with underlying chronic liver disease represent the majority of $\mathrm{HCC}$ cases. Mostly they result from viral hepatitis (HBV or HCV) infection or abuse of alcohol. However, the evolution in nonalcoholic fatty liver disease (NAFLD), which together with metabolic syndrome and obesity

El Lehleh et al., Afro-Egypt J Infect Endem Dis 2020;10(2):213-225

https://aeji.journals.ekb.eg/

http://mis.zu.edu.eg/ajied/home.aspx 
increases the hazard of liver cancer, will shorty become a main cause for liver cancer in Western countries [4].

Notably, about eighty percent of all HCC cases develop on top of liver cirrhosis. Consequently, comprehensive surveillance of all at risk populations by ultrasound is compulsory. hereafter, a considerable limitation of such standard method is its strong dependence on experience of operator as well as its poor sensitivity $(63 \%)$ for detection $\mathrm{HCC}$ at early stage [5]. In clinical practice, Alpha-fetoprotein (AFP) is the most commonly utilized serum marker for screening and initial HCC diagnosis, however, its sensitivity at a cut-off value 20 $\mathrm{ng} / \mathrm{mL}$ is approximately $60 \%$ and its specificity is indeed low [6]. Furthermore, AFP levels stick around its normal values in $15-30 \%$ of patients with advanced stage of HCC disease and may be elevated in some chronic hepatitis patients, patients with liver cirrhosis, and other liver diseases, leading to high rates of false negative and false-positive values [7]. Therefore, novel markers that complement the limitations of AFP are required for screening and more accurate diagnosis of $\mathrm{HCC}$

The TAM receptor tyrosine kinase (RTK) subfamily comprises three members: Axl, Tyro3, and Mer. Binding of the ligand Gas6 to Axl results in regulation of fundamental biological processes, including cell survival, motility and proliferation, via multiple downstream signaling pathways [8]. Soluble Axl (sAxl) that is measurable in the serum is resulted from proteolytic cleavage of Axl and in the release of the extracellular domain (ECD) [9]. It was previously reported that some malignancies showed aberrant expression of Axl and that this is critical for promoting cell proliferation, migration, angiogenesis and metastasis [10]. So, in the present work we aimed to evaluate diagnostic role of serum sAxl level as an HCC marker in comparison with serum Alphafetoprotein.

\section{PATIENTS AND METHODS}

This research was performed on 90 participants classified as 40 patients with hepatocellular carcinoma on top of liver cirrhosis, 30 cirrhotic patients in addition of 20 healthy participants of matched age and sex as controls. Participants were chosen from inpatient and outpatient clinic of Tropical Medicine and Oncology Departments, Menoufia University hospital, Menoufia, Egypt, in the period between May 2018 and July 2019. Patients with focal lesions in the liver other than HCC such as metastatic focal lesions, cholangiocarcinoma, hepatoblastoma, and hemangioma were excluded as well as patients with malignancy at other body sites.

Patients and controls were grouped in to the following: Group I: involved 40 patients with confirmed hepatocellular carcinoma on top of liver cirrhosis. Group II: comprised 30 liver cirrhosis patients in addition to Group III: Comprised 20 healthy participants as a controls. Hepatocellular carcinoma diagnosis was carried out by imaging studies (abdominal ultrasonography and confirmed by characteristic features in triphasic CT); and diagnosis of cirrhosis was carried out depending on the clinical examination, ultrasound finding together with laboratory investigations. Our study was executed in correspondence with the Declaration of Helsinki. An informed consent was provided by all participants before entering the study, and, the ethics Committee of the Faculty of Medicine, Menoufia University confirmed our research protocol.

For all participants, careful medical history and clinical examination were performed, in addition to laboratory investigations in the form of complete blood count, assessment of liver function tests (ALT, AST, serum albumin, INR, total and direct bilirubin), renal function tests, ESR and viral markers by ELISA (HCV Ab and HBV sAg) and confirmed by real time PCR. Serum AFP level was assessed by ELISA. Abdominal ultrasonography was performed for all participants and triphasic CT was done to assure hepatocellular carcinoma. HCC ppatients were subjected to baseline chest, abdomen and pelvis CT to determine distant metastases. Patients with HCC (GI) were classified according Barcelona Cancer of the Liver Clinic e (BCLC) [11]. Serum sAxl was quantitatively measured by ELISA. This kit was based on sandwich enzyme-linked immune-sorbent assay technology.

\section{Statistical analysis:}

Data was statistically analyzed using SPSS (statistical package for social science) (IBM, New York, USA) program version 22 for 
windows and for all the analysis a $\mathrm{p}$ value $<0.05$ was considered statistically significant.

\section{RESULTS}

This cross sectional study comprised 90 participants grouped into; group I included 40 HCC patients on top of liver cirrhosis with 27 males and 13 females $(67.5 \%$ and $32.5 \%$ respectively) with mean age $48.55 \pm 10.15$, group II included 30 patients with liver cirrhosis they were 18 males $(60.0 \%)$ and 12 females $(40 \%)$ and group III included 20 apparently healthy individuals. Statistical data analysis demonstrated matched sex and age $(p=0.722$, $\mathrm{p}=0.0 .086$, respectively) distributions among the three studied groups (Table 1). Regarding the clinical evaluation of studied groups anorexia and weight loss were more prevalent in HCC than in cirrhotic patients while we found no significant difference between the two patient groups concerning the history of abdominal pain, hematemesis, melena, jaundice, hepatic encephalopathy or edema LL (Table 2).

CBC finding showed that there was high statistical significant decrease in mean values of hemoglobin concentration and platelet count in HCC and cirrhotic groups compared to controls with no significant difference between GI and GII ( $p=0.341 \& 0.056$ respectively). However, we found that, there was non-significant difference between the three studied groups as regard white blood cell count $(\mathrm{p}=0.120)$ (Table 1).

Liver function tests in the three studied groups showed high significant difference between them, with significant increase in mean values of ALT, AST, total \& direct bilirubin and INR together with significant decrease in mean values of serum albumin in HCC and cirrhotic groups in comparison with control group, with no significant difference between cirrhosis and HCC patients as presented in table1. Regarding the etiology of cirrhosis Chronic HCV was the commonest etiology of cirrhosis in GI and GII $(82.5 \%$ and $86.7 \%$ respectively) patients (Table 2). Triphasic CT scan finding of HCC patients revealed that $77.5 \%$ of them had single hepatic focal lesion with mean size of focal lesion $5.44 \pm$ 3.39 (Table 3).

Table 1 showed that serum AFP and sAxl levels were significantly different between the three studied groups $(\mathrm{p}<0.001)$. sAxl and serum AFP were significantly higher in HCC group than in cirrhotic ( $p<0.001$ and $=0.047$ respectively) and control $(\mathrm{p}<0.001)$ groups as well as higher levels in cirrhotic patients than in controls $(\mathrm{p}<0.001)$ (Figure 2A \& 2B). Moreover, sAxl showed significantly higher levels in early HCC cases (Mean \pm SD was $83.98 \pm 21.45$ ) compared to cirrhotic group (Mean \pm SD was $58.88 \pm 64.26$ ) the $\mathrm{p}$ value was $<0.001$ in addition, higher levels in late (Mean \pm SD was $126.67 \pm 64.26$ ) than early cases but not statistically significant $(\mathrm{p}=$ 0.090). Regarding AFP, it was not significantly different between cirrhotic and early HCC patients.

Receiver operating characteristic curve (ROC curve) analysis revealed that AFP at cut off $>40.34$ has sensitivity, specificity and AUC (57.5\%, 60\% and 0.675 respectively), regarding sAxl, it has sensitivity $92.5 \%$, specificity $93.3 \%$ and AUC 0.949 at cut off value >63.5 in predicting HCC in cirrhotic patients. Moreover, the combination of AFP and sAxl at related cut off points showed higher sensitivity $(97.5 \%)$ and AUC 0.972 in predicting $\mathrm{HCC}$ in cirrhotic patients than one of them alone (Table 6 and Figure 2C \& 2D).

Correlation analysis between sAxl and patient features in the HCC and cirrhotic groups revealed that it was negatively related to platelet count $(\mathrm{r}=-0.342$ with $\mathrm{p}=0.031$ and $\mathrm{r}=-0.453$ with $p=0.012$ respectively) (Figure $1 \mathrm{C} \& 1 \mathrm{D}$ ), as well as significant positive association with Child score $(\mathrm{p}=0.006)$ and tumor size $(\mathrm{r}=0.403$ with $\mathrm{p}=0.010$ ) (Figure 1B) but not number of focal lesions in HCC group. However, serum AFP showed no significant association with tumor size (Figure 1A) or other studied parameters (Table $4 \& 5$ ). 
Table (1): Comparison between the three studied groups regarding demographic and laboratory parameters.

\begin{tabular}{|c|c|c|c|c|c|}
\hline & $\begin{array}{l}\text { Group I } \\
(n=40)\end{array}$ & $\begin{array}{l}\text { Group II } \\
(\mathbf{n}=\mathbf{3 0})\end{array}$ & $\begin{array}{c}\text { Group III } \\
(\mathbf{n}=\mathbf{2 0})\end{array}$ & $\begin{array}{l}\text { Test of } \\
\text { Sig. }\end{array}$ & $\mathbf{P}$ \\
\hline \multicolumn{6}{|l|}{ Sex } \\
\hline Male & $27(67.5 \%)$ & $18(60.0 \%)$ & $14(70.0 \%)$ & \multirow{2}{*}{$\chi^{2}=0.652$} & \multirow{2}{*}{0.722} \\
\hline Female & $13(32.5 \%)$ & $12(40.0 \%)$ & $6(30.0 \%)$ & & \\
\hline \multicolumn{6}{|l|}{ Age (years) } \\
\hline Mean \pm SD & $48.55 \pm 10.15$ & $54.20 \pm 12.85$ & $52.35 \pm 7.77$ & \multirow{2}{*}{$\mathrm{F}=2.520$} & \multirow{2}{*}{0.086} \\
\hline Median (Min. - Max.) & $48.0(32.0-70.0)$ & $51.0(33.0-77.0)$ & $51.0(40.0-69.0)$ & & \\
\hline \multicolumn{6}{|l|}{$\mathrm{Hb}(\mathrm{gm} / \mathrm{dl})$} \\
\hline Mean \pm SD. & $11.50 \pm 1.34$ & $11.0 \pm 1.87$ & $13.85 \pm 0.93$ & \multirow{2}{*}{$\mathrm{F}=24.691^{*}$} & \multirow{2}{*}{$<0.001^{*}$} \\
\hline Median (Min. - Max.) & $11.35(9.20-14.0)$ & $11.25(7.50-14.20)$ & $13.75(12.50-16.0)$ & & \\
\hline Sig. bet. Groups & \multicolumn{3}{|c|}{$\mathrm{p}_{1}=0.341, \mathrm{p}_{2}<0.001^{\prime \prime}, \mathrm{p}_{3}<0.001^{*}$} & & \\
\hline \multicolumn{6}{|l|}{ PLT $\left(\times 10^{3} / \mathrm{cm}^{3}\right)$} \\
\hline Mean \pm SD. & $141.8 \pm 66.30$ & $106.4 \pm 62.12$ & $232.6 \pm 42.71$ & \multirow{2}{*}{$\mathrm{H}=34.069^{*}$} & \multirow{2}{*}{$<0.001^{*}$} \\
\hline Median (Min. - Max.) & $127(71.0-372.0)$ & $96.0(31.0-222.0)$ & $225.0(150.0-300.0)$ & & \\
\hline Sig. bet Groups & \multicolumn{3}{|c|}{$\mathrm{p}_{1}=0.056, \mathrm{p}_{2}<0.001^{\prime \prime}, \mathrm{p}_{3}<0.001^{*}$} & & \\
\hline \multicolumn{6}{|l|}{ WBCs $\left(\times 10^{3} / \mathrm{cm}^{3}\right)$} \\
\hline Mean \pm SD. & $6.69 \pm 2.53$ & $5.45 \pm 1.69$ & $5.95 \pm 1.36$ & \multirow{2}{*}{$\mathrm{H}=4.233$} & \multirow{2}{*}{0.120} \\
\hline Median (Min. - Max.) & $6.55(2.90-11.30)$ & $5.0(2.20-9.80)$ & $6.0(4.0-9.0)$ & & \\
\hline \multicolumn{6}{|l|}{ ALT } \\
\hline Mean \pm SD. & $51.50 \pm 34.80$ & $38.27 \pm 21.83$ & $12.0 \pm 3.46$ & $\mathrm{H}=40647^{*}$ & $<0001^{*}$ \\
\hline Median (Min. - Max.) & $44.50(11.0-211.0)$ & $31.50(12.0-78.0)$ & $11.0(8.0-22.0)$ & $\mathrm{H}=40.64 /$ & $<0.001$ \\
\hline Sig. bet Groups & $\mathrm{p}_{1}=$ & $129, \mathrm{p}_{2}<0.001^{\prime \prime}, \mathrm{p}_{3}<0.0$ & & & \\
\hline AST & & & & & \\
\hline Mean \pm SD. & $53.10 \pm 27.67$ & $49.87 \pm 21.98$ & $13.45 \pm 1.36$ & $\mathrm{H}=42.767^{*}$ & $<0.001^{*}$ \\
\hline Median (Min. - Max.) & $58.50(10.0-117.0)$ & $50.0(18.0-97.0)$ & $13.0(11.0-16.0)$ & $\mathrm{H}=42.76 /$ & \\
\hline Sig. bet. Groups & $\mathrm{p}_{1}=$ & $854, \mathrm{p}_{2}<0.001^{\prime}, \mathrm{p}_{3}<0.0$ & & & \\
\hline Total bilirubin & & & & & \\
\hline Mean \pm SD. & $1.98 \pm 1.34$ & $2.57 \pm 2.44$ & $0.72 \pm 0.16$ & $H=35564^{*}$ & \\
\hline Median (Min. - Max.) & $1.40(0.50-5.50)$ & $1.55(0.50-8.20)$ & $0.70(0.50-1.0)$ & $H=35.564$ & $<0.001$ \\
\hline Sig. bet. Groups & $\mathrm{p}_{1}=$ & $.511, \mathrm{p}_{2}<0.001 " \mathrm{p}_{3}<0.0$ & & & \\
\hline Direct bilirubin & & & & & \\
\hline Mean \pm SD. & $0.77 \pm 0.71$ & $0.87 \pm 0.71$ & $0.16 \pm 0.07$ & & \\
\hline Median (Min. - Max.) & $0.50(0.10-2.70)$ & $0.68(0.10-2.50)$ & $0.10(0.10-0.30)$ & $\mathrm{H}=23.904$ & $<0.001$ \\
\hline Sig. bet Groups. & $\mathrm{p}_{1}=$ & $452, \mathrm{p}_{2}<0.001^{\prime}, \mathrm{p}_{3}<0.0$ & & & \\
\hline INR & & & & & \\
\hline Mean \pm SD & $1.30 \pm 0.29$ & $1.26 \pm 0.31$ & $1.0 \pm 0.0$ & $\mathrm{H}=35679^{*}$ & $<0001^{*}$ \\
\hline Median (Min. - Max.) & $1.0-1.90$ & $1.0-2.28$ & $1.0-1.0$ & & \\
\hline Sig. bet. Groups & $\mathrm{p}_{1}=$ & $.245, \mathrm{p}_{2}<0.001 "{ }^{\prime \prime} \mathrm{p}_{3}<0.0$ & & & \\
\hline Serum Albumin & & & & & \\
\hline Mean \pm SD & $2.94 \pm 0.61$ & $2.90 \pm 0.73$ & $4.57 \pm 0.32$ & $\mathrm{~F}-57177^{*}$ & $<0.001^{*}$ \\
\hline Median (Min. - Max.) & $1.90-4.20$ & $1.90-4.0$ & $4.0-5.0$ & & $<0.001$ \\
\hline Sig. bet. Groups & $\mathrm{p}_{1}=$ & $954, \mathrm{p}_{2}<0.001^{\prime \prime}, \mathrm{p}_{3}<0.0$ & & & \\
\hline Creatinin & & & & & \\
\hline Mean \pm SD. & $0.82 \pm 0.17$ & $0.90 \pm 0.15$ & $0.81 \pm 0.21$ & $\mathrm{H}=4543$ & 0103 \\
\hline Median (Min. - Max.) & $0.80(0.50-1.10)$ & $0.90(0.60-1.20)$ & $0.80(0.50-1.20)$ & $\mathrm{H}=4.543$ & 0.103 \\
\hline Alpha-fetoprotein & & & & & \\
\hline Mean \pm SD. & $410.2 \pm 787.0$ & $34.06 \pm 19.45$ & $5.58 \pm 2.17$ & $H=35675^{*}$ & $<0.001^{*}$ \\
\hline Median (Min. - Max.) & $60.0(1.50-3000.0)$ & $35.0(3.40-68.0)$ & $5.50(2.0-9.0)$ & & \\
\hline Sig. bet. Groups & $\mathrm{p}_{1}=$ & $047^{\prime \prime}, \mathrm{p}_{2}<0.001^{\prime \prime}, \mathrm{p}_{3}<0$. & & & \\
\hline SAXL & & & & & \\
\hline Mean \pm SD. & $108.5 \pm 54.55$ & $58.88 \pm 10.21$ & $29.47 \pm 8.39$ & $\mathrm{H}=68530^{*}$ & $<0.001^{*}$ \\
\hline Median (Min. - Max.) & $91.25(34.0-283.0)$ & $62.0(27.0-77.0)$ & $28.50(16.0-54.0)$ & $\Pi=00.550$ & -0.001 \\
\hline Sig. bet. Groups & $\mathrm{p}_{1}<$ & $001^{\prime \prime}, \mathrm{p}_{2}<0.001^{\prime \prime}, \mathrm{p}_{3}<0$. & & & \\
\hline ESR & & & & & \\
\hline Mean \pm SD. & $64.90 \pm 14.90$ & $15.67 \pm 12.75$ & $16.05 \pm 4.20$ & $\mathrm{~F}=$ & $<0001^{*}$ \\
\hline Median (Min. - Max.) & $67.0(12.0-87.0)$ & $12.50(5.0-55.0)$ & $16.50(10.0-22.0)$ & $169.859^{*}$ & -0.001 \\
\hline Sig. bet. Groups & $\mathrm{p}_{1}<$ & $.001^{\prime \prime}, \mathrm{p}_{2}<0.001^{\prime \prime}, \mathrm{p}_{3}=0$. & 94 & & \\
\hline
\end{tabular}

$\mathrm{Hb}$; hemoglobin concentration, WBCs; white blood cells, PLT; platelet count, INR; international normalized ratio, ALT; alanine aminotransferase; $\chi^{2}$ : Chi square test, $\mathrm{H}$ : H for Kruskal Wallis test, Pairwise comparison bet. each 2 groups was done using Post Hoc Test (Dunn's for multiple comparisons test), F: F for ANOVA test, Pairwise comparison bet. each 2 groups was done using Post Hoc Test (Tukey), p: $p$ value for comparing between the studied groups, $\mathrm{p}_{1}: \mathrm{p}$ value for comparing between Group I and Group II, $\mathrm{p}_{2}$ : $\mathrm{p}$ value for comparing between Group I and Group III, $\mathrm{p}_{3}$ : $\mathrm{p}$ value for comparing between Group II and Group III, *: Statistically significant at $p<0.05$.

El Lehleh et al., Afro-Egypt J Infect Endem Dis 2020;10(2):213-225

https://aeji.journals.ekb.eg/

http://mis.zu.edu.eg/ajied/home.aspx 
Table (2): Comparison between the two patient groups regarding clinical finding (history, examination and viral markers) $(\mathrm{n}=70)$.

\begin{tabular}{|c|c|c|c|c|}
\hline & $\begin{array}{l}\text { Group I } \\
(\mathbf{n}=\mathbf{4 0 )}\end{array}$ & $\begin{array}{c}\text { Group II } \\
(\mathbf{n}=\mathbf{3 0})\end{array}$ & Test of sig. & $\mathbf{p}$ \\
\hline \multicolumn{5}{|l|}{ History of :- } \\
\hline Anorexia & $22(55.0 \%)$ & $8(26.7 \%)$ & $\chi^{2}=5.619^{*}$ & $0.018^{*}$ \\
\hline Loss of weight & $20(50.0 \%)$ & $7(23.3 \%)$ & $\chi^{2}=5.145^{*}$ & $0.023^{*}$ \\
\hline Abdominal Pain & $11(27.5 \%)$ & $4(13.3 \%)$ & $\chi^{2}=2.043$ & 0.153 \\
\hline Hematemesis and/or melena & $4(10.0 \%)$ & $4(13.3 \%)$ & $\chi^{2}=0.188$ & 0.717 \\
\hline Jaundice & $8(20.0 \%)$ & $5(16.7 \%)$ & $\chi^{2}=0.126$ & 0.723 \\
\hline Encephalopathy & $3(7.5 \%)$ & $3(10.0 \%)$ & $\chi^{2}=0.137$ & 1.000 \\
\hline Edema lower limb & $16(40.0 \%)$ & $10(33.3 \%)$ & $\chi^{2}=0.326$ & 0.568 \\
\hline \multicolumn{5}{|l|}{ General Examination } \\
\hline Cachexia & $13(32.5 \%)$ & $5(16.7 \%)$ & $\chi^{2}=2.250$ & 0.134 \\
\hline Jaundice & $8(20.0 \%)$ & $5(16.7 \%)$ & $\chi^{2}=0.126$ & 0.723 \\
\hline Edema lower limb & $16(40.0 \%)$ & $10(33.3 \%)$ & $\chi^{2}=0.326$ & 0.568 \\
\hline \multicolumn{5}{|l|}{ Local Examination } \\
\hline \multicolumn{5}{|l|}{ Ascites } \\
\hline No & $20(50.0 \%)$ & $18(60.0 \%)$ & \multirow{3}{*}{$\chi^{2}=0.700$} & \multirow{3}{*}{0.705} \\
\hline Mild to moderate & $13(32.5 \%)$ & $8(26.7 \%)$ & & \\
\hline Massive & $7(17.5 \%)$ & $4(13.3 \%)$ & & \\
\hline Umbilical hernia & $3(7.5 \%)$ & $5(16.7 \%)$ & $\chi^{2}=1.423$ & 0.275 \\
\hline \multicolumn{5}{|l|}{ Examination of liver } \\
\hline No hepatomegaly & $31(77.5 \%)$ & $26(86.7 \%)$ & \multirow{2}{*}{$\chi^{2}=0.953$} & \multirow{2}{*}{0.329} \\
\hline Hepatomegaly & $9(22.5 \%)$ & $4(13.3 \%)$ & & \\
\hline \multicolumn{5}{|l|}{ Examination of spleen } \\
\hline Surgically removed & $3(7.5 \%)$ & $0(0 \%)$ & \multirow{3}{*}{$\chi^{2}=4.214$} & \multirow{3}{*}{0.146} \\
\hline No splenomegaly & $8(20.0 \%)$ & $11(36.7 \%)$ & & \\
\hline Splenomegaly & $29(72.5 \%)$ & $19(63.3 \%)$ & & \\
\hline \multicolumn{5}{|l|}{ Viral markers } \\
\hline HCV-Ab & $33(82.5 \%)$ & $26(86.7 \%)$ & \multirow{3}{*}{$\chi^{2}=0.368$} & \multirow{3}{*}{1.000} \\
\hline HBVs Ag & $5(12.5 \%)$ & $3(10.0 \%)$ & & \\
\hline $\mathrm{HCV}-\mathrm{Ab}+\mathrm{HBVs} \mathrm{Ag}$ & $2(5.0 \%)$ & $1(3.3 \%)$ & & \\
\hline Child-Pugh score & $7(5.0-12.0)$ & $7(5.0-14.0)$ & $\mathrm{U}=586.50$ & 0.870 \\
\hline $\mathrm{A}$ & $19(47.5 \%)$ & $14(46.7 \%)$ & \multirow{3}{*}{$\chi^{2}=0600$} & \multirow{3}{*}{0.971} \\
\hline $\mathrm{B}$ & $10(25.0 \%)$ & $7(23.3 \%)$ & & \\
\hline $\mathrm{C}$ & $11(27.5 \%)$ & $9(30.0 \%)$ & & \\
\hline
\end{tabular}

\footnotetext{
$\chi^{2}$ : Chi square test $\mathrm{U}$ : Mann Whitney test,

$\mathrm{p}$ : $\mathrm{p}$ value for comparing between the studied groups *: Statistically significant at $\mathrm{p} \leq 0.05$
} 
Table (3): Comparison between the studied patient groups regarding abdominal CT finding $(\mathrm{n}=70)$.

\begin{tabular}{|c|c|c|c|}
\hline & & \multicolumn{2}{|c|}{ CT } \\
\hline & & $\begin{array}{l}\text { Group I } \\
(n=40)\end{array}$ & $\begin{array}{c}\text { Group II } \\
(\mathbf{n}=\mathbf{3 0})\end{array}$ \\
\hline \multicolumn{4}{|l|}{ Liver } \\
\hline 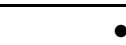 & Enlarged & $9(22.5 \%)$ & $4(13.3 \%)$ \\
\hline$\bullet$ & Not enlarged & $31(77.5 \%)$ & $26(86.7 \%)$ \\
\hline$\chi^{2}(\mathbf{p})$ & & \multicolumn{2}{|c|}{$0.953(0.329)$} \\
\hline \multicolumn{4}{|c|}{\begin{tabular}{|l|} 
Focal lesion \\
Number \\
\end{tabular}} \\
\hline$\bullet$ & Single & $31(77.5 \%)$ & - \\
\hline \multirow{2}{*}{\multicolumn{2}{|c|}{ - $\quad$ Two or more }} & $9(22.5 \%)$ & - \\
\hline & & \multicolumn{2}{|c|}{-} \\
\hline \multicolumn{4}{|c|}{ Size (cm) } \\
\hline Mean $\pm s$ & & $5.44 \pm 3.39$ & - \\
\hline Median & in. - Max.) & $4.30(1.0-11.5)$ & - \\
\hline \multicolumn{4}{|c|}{$\begin{array}{l}\text { Spleen } \\
\text { Spleen size : }\end{array}$} \\
\hline$\bullet$ & Surgically removed & $3(7.5 \%)$ & $0(0 \%)$ \\
\hline - & Not enlarged & $8(20.0 \%)$ & $11(36.7 \%)$ \\
\hline - & Enlarged & $29(72.5 \%)$ & $19(63.3 \%)$ \\
\hline$\chi^{2}(\mathbf{p})$ & & \multicolumn{2}{|c|}{$5.773(0.144)$} \\
\hline \multicolumn{4}{|c|}{ Splenic collaterals : } \\
\hline$\bullet$ & Yes & $9(22.5 \%)$ & $6(20.0 \%)$ \\
\hline$\chi^{2}(\mathbf{p})$ & & \multicolumn{2}{|c|}{$064(0.801)$} \\
\hline \multicolumn{4}{|c|}{$\begin{array}{l}\text { Portal vein } \\
\text { PV Size: }\end{array}$} \\
\hline$\bullet$ & Not dilated & $13(32.5 \%)$ & $14(46.7 \%)$ \\
\hline$\bullet$ & Dilated & $27(67.5 \%)$ & $16(53.3 \%)$ \\
\hline$\chi^{2}(\mathbf{p})$ & & \multicolumn{2}{|c|}{$\frac{1}{1.452(0.228)}$} \\
\hline \multicolumn{4}{|c|}{ PV thrombosis: } \\
\hline$\bullet$ & Patent PV & $34(85.0 \%)$ & $30(100.0 \%)$ \\
\hline - & PV Thrombosis & $6(15.0 \%)$ & $0(0.0 \%)$ \\
\hline$\chi^{2}(\mathbf{p})$ & & \multicolumn{2}{|c|}{$4.922^{*}\left(0.034^{*}\right)$} \\
\hline \multicolumn{4}{|l|}{ Ascites: } \\
\hline$\bullet$ & No & $20(50.0 \%)$ & $18(60.0 \%)$ \\
\hline - & Mild & $9(22.5 \%)$ & $3(10.0 \%)$ \\
\hline - & Moderate & $4(10.0 \%)$ & $5(16.7 \%)$ \\
\hline - & Massive & $7(17.5 \%)$ & $4(13.3) \%$ \\
\hline$\chi^{2}(\mathbf{p})$ & & \multicolumn{2}{|c|}{$2.626(0.470)$} \\
\hline
\end{tabular}

\section{$\chi^{2}$ : Chi square test}

$\mathrm{p}: \mathrm{p}$ value for comparing between the studied groups, *: Statistically significant at $\mathrm{p}<0.05$ 
Table (4): Correlation between AFP and SAXL with age and laboratory parameters in group I and group II.

\begin{tabular}{|c|c|c|c|c|c|}
\hline \multirow{2}{*}{\multicolumn{2}{c|}{}} & \multicolumn{2}{c|}{ AFP } & \multicolumn{2}{c|}{ SAXL } \\
\cline { 3 - 6 } \multirow{2}{*}{ Age } & $\mathbf{r}_{\mathbf{s}}$ & -0.069 & -0.126 & 0.077 & 0.277 \\
\cline { 2 - 6 } & $\mathbf{P}$ & 0.670 & 0.508 & 0.638 & 0.139 \\
\hline \multirow{2}{*}{ Hb } & $\mathbf{r}_{\mathbf{s}}$ & 0.176 & -0.197 & 0.047 & 0.242 \\
\cline { 2 - 6 } & $\mathbf{P}$ & 0.278 & 0.297 & 0.775 & 0.199 \\
\hline \multirow{2}{*}{ PLT } & $\mathbf{r}_{\mathbf{s}}$ & 0.255 & 0.354 & -0.342 & -0.453 \\
\cline { 2 - 6 } & $\mathbf{P}$ & 0.112 & 0.055 & $0.031^{*}$ & $0.012^{*}$ \\
\hline \multirow{2}{*}{ WBCs } & $\mathbf{r}_{\mathbf{s}}$ & -0.030 & 0.130 & 0.062 & 0.199 \\
\cline { 2 - 6 } & $\mathbf{P}$ & 0.855 & 0.493 & 0.703 & 0.293 \\
\hline \multirow{2}{*}{ ALT } & $\mathbf{r}_{\mathbf{s}}$ & -0.253 & -0.023 & -0.176 & 0.033 \\
\cline { 2 - 6 } & $\mathbf{P}$ & 0.115 & 0.905 & 0.277 & 0.864 \\
\hline \multirow{2}{*}{ Total bilirubin } & $\mathbf{r}_{\mathbf{s}}$ & 0.055 & 0.137 & -0.145 & 0.015 \\
\cline { 2 - 6 } & $\mathbf{P}$ & 0.734 & 0.472 & 0.373 & 0.939 \\
\hline \multirow{2}{*}{ INR } & $\mathbf{r}_{\mathbf{s}}$ & 0.006 & -0.013 & -0.147 & -0.085 \\
\cline { 2 - 6 } & $\mathbf{P}$ & 0.971 & 0.945 & 0.367 & 0.655 \\
\hline \multirow{2}{*}{ Albumin } & $\mathbf{r}_{\mathbf{s}}$ & 0.156 & 0.055 & 0.266 & -0.123 \\
\cline { 2 - 6 } & $\mathbf{P}$ & 0.335 & 0.773 & 0.097 & 0.517 \\
\hline \multirow{2}{*}{ Tumour size (cm) } & $\mathbf{r}_{\mathbf{s}}$ & -0.004 & - & 0.403 & - \\
\cline { 2 - 6 } & $\mathbf{P}$ & 0.982 & - & 0.010 & - \\
\hline \multirow{2}{*}{ AFP } & $\mathbf{r}_{\mathbf{s}}$ & - & - & -0.223 & -0.088 \\
\cline { 2 - 6 } & $\mathbf{P}$ & - & - & 0.167 & 0.642 \\
\hline
\end{tabular}

$\mathrm{Hb}$; hemoglobin concentration, WBCs; white blood cells, PLT; platelet count, INR; international normalized ratio, ALT; alanine aminotransferase; $\mathbf{r}_{\mathbf{s}}$ : Spearman coefficient, $*$ : Statistically significant at $p<0.05$

Table (5): Relation between AFP and SAXL with Child-Pugh score, number of focal lesion (s) and portal vein thrombosis in group I.

\begin{tabular}{|c|c|c|c|c|c|}
\hline & \multirow{2}{*}{$\mathbf{N}$} & \multicolumn{2}{|l|}{ AFP } & \multicolumn{2}{|c|}{ SAXL } \\
\hline & & Median (Min. - Max.) & Mean \pm SD. & Median (Min. - Max.) & Mean \pm SD. \\
\hline \multicolumn{6}{|l|}{ Child } \\
\hline $\mathrm{A}$ & 19 & $115.0(4.30-2451.0)$ & $438.85 \pm 668.67$ & $82.0(34.0-116.0)$ & $82.77 \pm 19.06$ \\
\hline $\mathrm{B}$ & 10 & $30.0(7.20-60.0)$ & $32.48 \pm 18.63$ & $87.0(75.0-208.0)$ & $102.80 \pm 40.51$ \\
\hline $\mathrm{C}$ & 11 & $91.90(1.50-3000.0)$ & $704.09 \pm 1170.91$ & $141.0(0.0-283.0)$ & $158.23 \pm 73.91$ \\
\hline $\mathbf{H}(\mathbf{p})$ & & \multicolumn{2}{|c|}{$5.129(0.077)$} & \multicolumn{2}{|c|}{$10.107(0.006 *)$} \\
\hline \multicolumn{6}{|l|}{ Number } \\
\hline One & 31 & $60.0(1.50-3000.0)$ & $375.40 \pm 787.65$ & $97.0(0.0-283.0)$ & $283.0 \pm 115.89$ \\
\hline Two or more & 9 & $107.0(6.0-2451.0)$ & $530.05 \pm 819.68$ & $82.0(0.0-283.0)$ & $83.17 \pm 16.84$ \\
\hline $\mathbf{U}(\mathbf{p})$ & & \multicolumn{2}{|c|}{$113.0(0.406)$} & \multicolumn{2}{|c|}{$\begin{array}{l}85.50(0.080) \\
\end{array}$} \\
\hline \multicolumn{6}{|l|}{ PV thrombosis } \\
\hline Patent PV & 34 & $60.0(4.30-3000.0)$ & $387.01 \pm 712.80$ & $91.25(0.0-283.0)$ & $106.65 \pm 49.75$ \\
\hline PV Thrombosis & 6 & $63.50(1.50-3000.0)$ & $541.58 \pm 1205.42$ & $91.0(61.0-283.0)$ & $119.17 \pm 81.92$ \\
\hline $\mathbf{U}(\mathbf{p})$ & & \multicolumn{2}{|c|}{$85.50(0.541)$} & \multicolumn{2}{|c|}{$100.50(0.956)$} \\
\hline
\end{tabular}

\section{U: Mann Whitney test $\quad$ H: H for Kruskal Wallis test}

p: $p$ value for association between AFP and sAxl with different parameters

*: Statistically significant at $p<0.05$ 
Table (6): Agreement (sensitivity, specificity) of AFP and sAXL and their combination in diagnosis of hepatocellular carcinoma vs liver cirrhosis.

\begin{tabular}{|c|c|c|c|c|c|c|c|c|}
\hline & AUC & $\mathbf{p}$ & C.I & 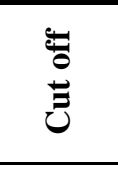 & 㺼 & 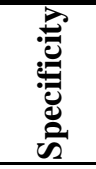 & $\overrightarrow{2}$ & $\vec{z}$ \\
\hline AFP & 0.675 & $0.013^{*}$ & $0.547-0.803$ & $>40.34$ & 57.50 & 60.0 & 65.7 & 51.4 \\
\hline sAXL & 0.949 & $<0.001^{*}$ & $0.892-1.00$ & $>63.5$ & 92.50 & 93.33 & 94.9 & 90.3 \\
\hline AFP + SAXL & 0.972 & $<0.001 *$ & $0.901-0.997$ & $\begin{array}{c}>40.34 \\
+ \\
>63.5\end{array}$ & 97.50 & 93.33 & 95.1 & 96.6 \\
\hline
\end{tabular}

AFP; Alpha-fetoprotein, sAxl; Soluble Axl, AUC; area under the curve, PPV; positive predictive value, NPV; Negative predictive value
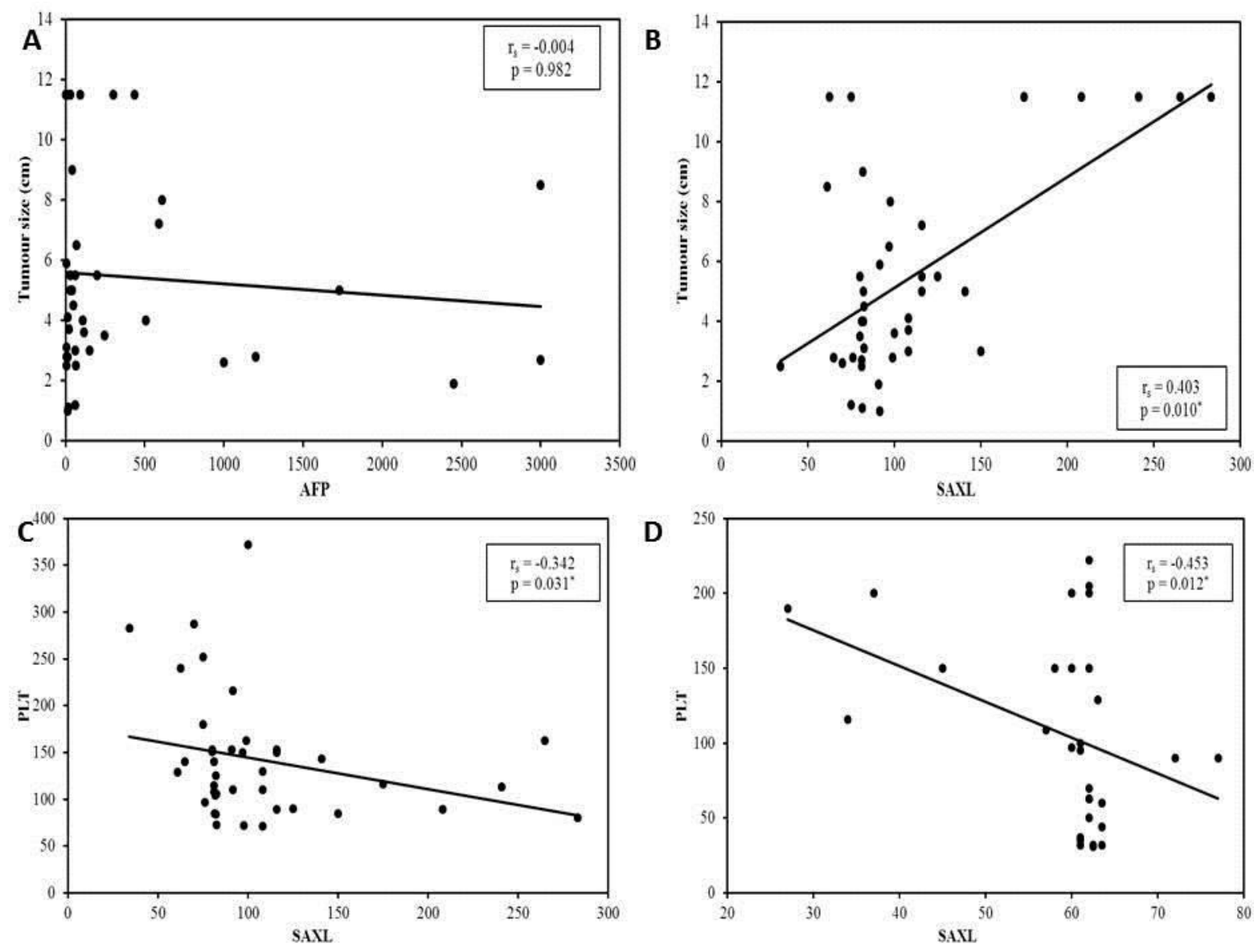

Figure 1: (A): Correlation between AFP and tumor size in GI, (B): Correlation between sAxl and tumor size in GI, (C): Correlation between sAxl and platelet count in GI, (D): Correlation between sAxl and platelet count in GII. 

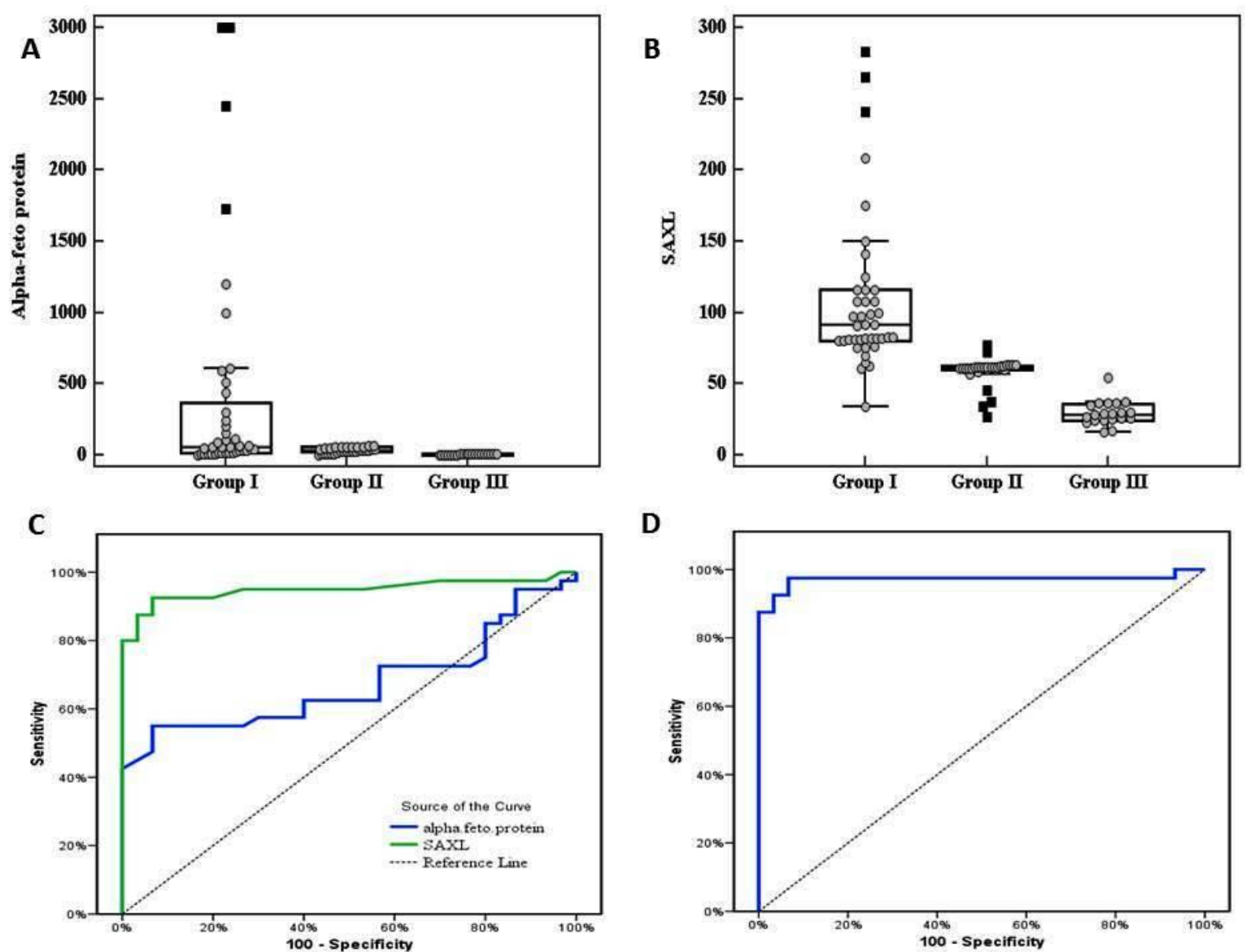

Figure 2: ((A): AFP levels in studied groups, (B): sAxl levels in studied groups, (C): ROC curve of AFP and sAxl for diagnosis of HCC in cirrhotic patients, (D): ROC curve of combined AFP and sAxl for diagnosis of $\mathrm{HCC}$ in cirrhotic patients

\section{DISCUSSION}

HCC represented the fourth primary trigger of cancer-related mortality in the world. Currently $\mathrm{HCC}$ is the eighth leading cause of cancer death in females and the fifth leading cause of death in males in the United States [12].

As most patients with $\mathrm{HCC}$ are discovered late with underlying liver dysfunction, the mortality rate of $\mathrm{HCC}$ is comparable with its incidence rate. Early discovery of HCC is subsequently quite important to improve the survival of these patients. The keys for efficient treatment of patients with $\mathrm{HCC}$ are the early diagnosis and treatment of cases since HCC is amongst the cancers with the worst prognosis. Using of valuable biological markers for patients who are at risk for HCC may help lowering HCC mortality and decrease medical costs [13].

The most widely used biomarker in HCC diagnosis is AFP, however its clinical value is challenged due to low sensitivity and specificity
[6]. Other serum biomarkers are being actively evaluated including Golgi protein 73, glypican-3, Des-gamma carboxyprothrombin and others; however, none of them have been adequately explored to be recommended as a screening test for HCC [14].

Feneyrolles and his colleagues [15] has reported that Axl is a member of the TAM (Tyro3, Axl, Mer) subfamily of receptor tyrosine kinases and so far known to be involved in cancer development and mediation of chemoresistance. Axl activation is involved in diverse biologic cell responses including cell proliferation, motility and survival in some malignancies.

The extracellular domains of the TAM receptors are formed mainly from two structural modules which are repeatedly utilized in other RTK ectodomains, however that are configured in the TAMs in a defining two-plus-two combination. It was found that ectodomains' aminoterminal regions bear tandem immunoglobulin- 
related domains that mediate ligand binding are followed by tandem type III repeats of fibronectin [16]. All three TAM receptors has a catalytically eligible protein-tyrosine kinase and all have a single transmembrane domain. In the complete vertebrate "kinome," the TAMs are closely linked to Ron, the macrophagestimulating protein receptor and Met, the hepatocyte growth factor receptor [17]. AXL that is a cell surface receptor tyrosine kinase [18], is proteolytically cleaved leading to the release of the extracellular domain that is known as soluble Axl (sAxl), and can be checked in the serum [9]. This work aimed to evaluate the role of sAxl levels for diagnosis of HCC developed on top of liver cirrhosis in comparison with serum AFP in addition to evaluate the combination of sAxl and serum AFP in diagnosis of hepatocellular carcinoma.

In the present study, Chronic HCV was the commonest etiology of liver disease in cirrhotic and hepatocellular carcinoma patients representing $82.5 \%$ and $86.7 \%$ respectively, this agreed with Sabry et al. and Amer et al. [19 and 20] who reported that in Egypt, chronic hepatitis $\mathrm{C}$ constituted the main risk factor for cirrhosis and the subsequent development of HCC.

Chronic hepatitis B and C in addition to cirrhosis irrespective of its etiology are considered the risk factors predisposing for HCC. Throughout Europe, North America and other low prevalence regions, the majority of patients have underlying cirrhosis unrelated to $\mathrm{HCV}$ or $\mathrm{HBV}$ infection [21]. The etiological role of hepatitis viral infection as a hazard for development of HCC is different worldwide and is related to the prevalence of viral infection as well as other reasons for liver cirrhosis. Kumar et al. [22] previously documented that in China, chronic hepatitis B virus is the main factor contributing for HCC development.

The present study showed higher AFP levels in both patients with liver cirrhosis and those with HCC than in healthy participants in addition to significantly higher values in HCC patients compared to cirrhotic patients. The level of AFP for diagnosis of HCC cases was changeable in different researches. Yoshida et al. [23] found normal AFP values in approximately one-third of HCC cases in addition, many patients with HCC have AFP levels less than $400 \mathrm{ng} / \mathrm{mL}$, so it troublesome to be used for diagnosis of HCC. Also, Tsai and his colleagues [24] observed that at least one third of patients with small hepatocellular carcinoma and about $30 \%$ of those with advanced HCC cases will be missed except if other diagnostic tools are performed. Also they found that AFP levels in some non-malignant liver diseases may also be elevated. Consequently, it is evident that AFP alone is not a valid predictor for HCC.

In our study, we observed that the values of sAxl in HCC group was significantly increased compared to other groups and in cirrhotic group in comparison with control group. sAxl in HCC group ranged $34.0-283.0 \mathrm{ng} / \mathrm{ml}(\mathrm{X} \pm \mathrm{SD}$ were 108.5 \pm 54.55$)$ and in cirrhotic group ranged 27$77 \mathrm{ng} / \mathrm{ml}(\mathrm{X} \pm \mathrm{SD}$ were $58.88 \pm 10.21)$ while in control group it was $16.0-54.0 \mathrm{ng} / \mathrm{ml}(\mathrm{X} \pm \mathrm{SD}$

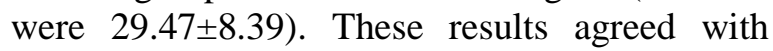
Dengler et al. [25] who found high levels of sAxl in cirrhosis than healthy controls and higher levels in HCC on top of cirrhosis compared to health controls and also compared to cirrhotic patients. Similarly, Reichl et al. [26] reported that levels sAxl in patients with HCC (most patients were related to HBV) were significantly higher than in healthy participants and patients with liver cirrhosis furthermore, it was higher in cirrhotic patients than healthy controls but this difference was statistically insignificant. We propose that the discrepancy between this results and ours could be attributed to different study population with different etiology of liver disease in the 2 studies.

In liver fibrosis, it was found that the gathering of extracellular matrix components resulted from activation of hepatic stellate cells (HSC), induces an environment permissive for development of tumor. During chronic liver injury the HSCs transform from a quiescent state into a myofbroblast-like phenotype, that proliferate and migrate to the areas of necrosis and regeneration [27]. In a study done by Barcena et al. [28], they observed that HSCs acquired from wild type as well as Axl-/- mice handled by recombinant Gas6, Axl siRNAs or the Axl inhibitor BGB324, the Gas6/AXL axis was needed for activation of HSC. Moreover, Dengler et al. [25] found that serum Axl levels increased in parallel to advancement of chronic liver disease and the evolution of cirrhosis independent of liver disease etiology. Okabe et al. [29] documented that during development of fibrosis there was increased proteolysis of Axl in myofibroblasts that facilitate an $\mathrm{HCC}$ permissive state. 
In the current study ROC analysis for AFP showed that AUC was 0.675 at cut off point $40.34 \mathrm{ng} / \mathrm{ml}$, the sensitivity and specificity were (57.50\% and $60.0 \%$ respectively) for detection of HCC in cirrhotic patients. Trevisani et al. [30] documented that specificity of AFP varies from $76 \%$ to $96 \%$ and could be increased with elevated cutoff values used but with lowering specificity. Sabry et al. [19] also has reported that at cut off point $55 \mathrm{ng} / \mathrm{ml}$ AFP had sensitivity $81.3 \%$ and its specificity was $70.0 \%$, moreover, Omran et al. [31] reported that AFP at a cut-off level $6.5 \mathrm{ng} / \mathrm{ml}$ the AUROC was 0.611 with a sensitivity $63 \%$ and specificity of $43 \%$.

Regarding sAxl, AUROC in our study was 0.949 with sensitivity $92.5 \%$ and specificity $93.33 \%$ at cut off point $63.5 \mathrm{ng} / \mathrm{l}$ with increased sensitivity up to $97.5 \%$ with combined use of both AFP and sAxl suggesting the remarkable role of sAxl in diagnosis of HCC. Previous reports supported our findings where Dengler et al. [25] postulated that, sAxl is up-regulated in patients with HCC and that sAxl at a cut-off point $49.71 \mathrm{ng} / \mathrm{mL}$ had a sensitivity and specificity of $83.3 \%$ and $86.7 \%$, respectively, moreover. Reichl et al. [26] reported that the combined assessment of sAxl and AFP can additionally improve the sensitivity for the detection of HCC up to $88.5 \%$.

Furthermore, when comparing between sAxl levels in early HCC and cirrhotic patients we observed significantly higher levels of sAxl in patients with early HCC than cirrhotic patients that indicates the role of $\mathrm{sAxl}$ for early prediction and diagnosis of HCC and could be used for screening of HCC in cirrhotic patients. Previous report supported our findings where Reichl et al. [26] found that serum levels of sAxl were significantly higher in patients with early HCC versus patients with cirrhosis or chronic liver disease. However, a multicenter study found that sAxl levels were obviously elevated in patients with both early and late stages of HCC but without significant difference between cirrhotic patients and patients with early stage HCC [25].

The findings of a correlation analysis of serum sAxl and tumor size showed a clear significant positive association with significantly higher levels of sAxl with larger tumor size. It has previously been reported by $\mathrm{Xu}$ and his colleagues [32] that sAxl join differentially to a soluble ligand that is known as growth arrest signal 6 (Gas-6), and intercedes its functions intracellularly through activation of phosphatidyl-inositol-3 kinase (PI3K)/Akt14 and, to a lesser extent, ERK-p38 mitogenactivated protein kinase (MAPK). Former mechanistic evidence has elucidated the link of Axl in HCC progression by recognizing Axl as a downstream organizer of the Hippo signaling pathway, a key regulator for development of tissues, whose disruption is involved in tumor cell invasion, proliferation and migration via activation of MAPK.

It was proposed that, in $\mathrm{HCC}$ cases $\mathrm{Axl}$ is a hopeful therapeutic target. The R428 had antiproliferative effects within the low micromolar range in a wide array of immortalized HCC cell lines. The incremental effects of $\mathrm{R} 428$ on sorafenib-induced growth inhibitory as well as pro-apoptotic possibility, are hopeful lineaments for the evolution of Axl-inhibitors in the firstline management of patients with advanced endeavoring to prolong progression-free survival until patients are faced untreatable stages due to liver dysfunctions [33].

\section{CONCLUSION}

sAXL is a valuable marker for early detection of hepatocellular carcinoma in liver cirrhosis patients. sAxl outperforms AFP in detecting $\mathrm{HCC}$, especially in early $\mathrm{HCC}$ and in AFPnegative HCC. The accuracy, sensitivity and negative predictive value for diagnosis of hepatocellular carcinoma in cirrhotic patients could be increased to $93.33 \%, 97.50 \%$, and $96.6 \%$ respectively with combined assay of both serum AFP and sAXL at cut off points ( $>40.34$ $\mathrm{ng} / \mathrm{mL}$ and $>63.5 \mathrm{ng} / \mathrm{mL}$ respectively). Furthermore, sAxL significantly increased with increasing tumor size.

\section{List of Abbreviations}

Hepatocellular carcinoma (HCC), nonalcoholic fatty liver disease (NAFLD), ultrasound (US), Alpha-fetoprotein (AFP), Receptor tyrosine kinase (RTK) Extracellular domain (ECD), soluble Axl (sAxl), Extracellular domain (ECD), Barcelona Cancer of the Liver Clinic e (BCLC) Enzyme linked immunosorbent assay (ELISA), SPSS (statistical package for social science), Receiver operating characteristic curve (ROC curve) Des-gamma carboxyprothrombin (DCP), Golgi protein 73(GP73), receptor tyrosine kinase (RTK), growth arrest signal 6 (Gas-6), mitogenactivated protein kinase (MAPK), phosphatidylinositol-3 kinase (PI3K) . 
Funding: No funding resources.

Conflict of Interest: The authors declare that there was no conflict of interest.

\section{Ethical consideration}

Our study was executed in correspondence with the Declaration of Helsinki. An informed consent was provided by all participants before entering the study, and, the ethics Committee of the Faculty of Medicine, Menoufia University confirmed our research protocol.

\section{REFERENCES}

1. Jemal A, Ward EM, Johnson CJ, Cronin KA, Ma $\mathrm{J}$, Ryerson B, et al. Annual report to the nation on the status of cancer, 1975-2014, featuring survival. J Natl Cancer Inst 2017; 109(9).

2. Ikai I, Kudo M, Arii S, Omata M, Kojiro M, Sakamoto M, et al. Report of the 18th follow-up survey of primary liver cancer in Japan. Hepatol Res 2010; 40:1043-1059.

3. Bruix J, Sherman M. American Association for the Study of Liver Diseases. Management of hepatocellular carcinoma: an update. Hepatology 2011; 53:1020-1022.

4. Younossi Z, Stepanova M, Ong JP, Jacobson IM, Bugianesi E, Duseja A, et al. Nonalcoholic steatohepatitis is the fastest growing cause of hepatocellular carcinoma in liver transplant candidates. Clin Gastroenterol Hepatol 2019; 17(4):748- 755.

5. Singal A, Volk ML, Waljee A, Salgia R, Higgins P, Rogers MA, et al. Meta-analysis: surveillance with ultrasound for early-stage hepatocellular carcinoma in patients with cirrhosis. Aliment Pharmacol Ther 2009; 30:37-47.

6. Jang ES, Jeong SH, Kim JW, Choi YS, Leissner $\mathrm{P}$, Brechot C, et al. Diagnostic performance of alpha-fetoprotein, protein induced by vitamin $\mathrm{K}$ absence, Osteopontin, Dickkopf-1 and its combinations for hepatocellular carcinoma. PLoS One 2016; 11: e0151069.

7. Jing W, Luo P, Zhu M, Ai Q, Chai H, Tu J, et al. Prognostic and diagnostic significance of SDPRCavin-2 in hepatocellular carcinoma. Cell Physiol Biochem 2016; 39:950-60.

8. Graham DK, DeRyckere D, Davies KD, Earp HS: The TAM family: phosphatidylserine sensing receptor tyrosine kinases gone awry in cancer. Nat Rev Cancer 2014;14(12):769-785.
9. Liu X, Gong Y, Jia J, Bai Y, Gui S, Wang T, et al. Plasma concentrations of sAxl are associated with severe preeclampsia. Clin Biochem 2014; 47:173-176.

10. Maacha S, Hong J, von Lersner A, Zijlstra A, Belkhiri A. AXL mediates esophageal adenocarcinoma cell invasion through regulation of extracellular acidification and lysosome trafficking. Neoplasia 2018; 20(10): 1008-1022.

11. Pinato DJ, Sharma R, Allara E, Yen C, Arizumi $\mathrm{T}$, Kubota $\mathrm{K}$ et al. The ALBI grade provides objective hepatic reserve estimation across each BCLC stage of hepatocellular carcinoma. $J$ Hepatol 2017; 66(2):338-346.

12. Siegel RL, Miller KD, Jemal A. Cancer statistics, 2018. CA Cancer J Clin 2018; 68:730.

13. Khalaf N, Ying J, Mittal S, Temple S, Kanwal F, Davila J, et al. Natural history of untreated hepatocellular carcinoma in a US cohort and the role of cancer surveillance. Clin Gastroenterol Hepatol 2017; 15:273-281.

14. Clark P. Serum markers for hepatocellular carcinoma. Clin Liver Dis 2016; 8(2):29-33.

15. Feneyrolles, Spenlinhauer A, Guiet L, Fauvel B, Daydé-Cazals B, et al. Axl kinase as a key target for oncology: focus on small molecule inhibitors. Mol Cancer Ther 2014; 13: 21412148.

16. Lew ED, Oh J, Burrola PG, Lax I, Zagórska A, Través PG, et al. Differential TAM receptorligand-phospholipid interactions delimit differential TAM bioactivities. e Life 2014;3: e03385.

17. Manning G, Whyte DB, Martinez R, Hunter T, Sudarsanam S. The protein kinase complement of the human genome. Science 2002; 298(5600):1912-34.

18. Wu X, Liu X, Koul S, Lee CY, Zhang Z and Halmos B. AXL kinase as a novel target for cancer therapy. Oncotarget 2014; 5(20):954663.

19. Sabry HS, Mohamed HI, Radwan WM, El Abd NS. Study of Serum Golgi Protein 73 Level as a Marker for Diagnosis of Hepatocellular Carcinoma. Afro-Egypt $J$ Infect Endem Dis 2015; 5(3), Page 160-170.

20. Amer, Nehad A, Gemaay and Mohamed A. Prevalence of viral hepatitis in Egyptian patients with hepatocellular carcinoma. Egyptian Liver Journal 2013; 3 (1): 6-9.

21. Constantin CV, Streba CT, Rogoveanu I, NitaStefanescu L, Ionescu AG. Cirrhosis and chronic

El Lehleh et al., Afro-Egypt J Infect Endem Dis 2020;10(2):213-225

https://aeji.journals.ekb.eg/

http://mis.zu.edu.eg/ajied/home.aspx 
viral hepatitis as risk factors for hepatocellular carcinoma: Romanian single clinic experience. Maedica (Buchar) 2010; 5:265-270.

22. Kumar M, Kumar R, Hissar SS, Saraswat MK, Sharma BC, Sakhuja P, et al. Risk factors analysis for hepatocellular carcinoma in patients with and without cirrhosis: a case-control study of 213 hepatocellular carcinoma patients from India. J Gastroenterol Hepatol 2007; 22: 11041111.

23. Yoshida S, Kurokohchi K, Arima K, Masaki T, Hosomi N, Funaki T, et al. Clinical significance of Lens culinaris agglutinin-reactive fraction of serum alpha-fetoprotein in patients with hepatocellular carcinoma. Int J Oncol 2002; 20: 305-309.

24. Tsai JF, Jeng JE, Chuang LY, You HL, Ho MS, Lai CS, et al. Serum insulin-like growth factorII and alpha-fetoprotein as tumor markers of hepatocellular carcinoma. Tumour Biol 2003; 24(6): 291-8.

25. Dengler $M$, Staufer K, Huber H, Stauber R, Bantel H, Weiss KH, et al. Soluble Axl is an accurate biomarker of cirrhosis and hepatocellular carcinoma development: results from a large scale multicenter analysis. Oncotarget 2017; 8(28): 46234-46248.

26. Reichl P, Fang M, Starlinger P, Staufer K, Nenutil R, Muller P, et al. Multicenter analysis of soluble Axl reveals diagnostic value for very early stage hepatocellular carcinoma. Int $J$ Cancer 2015; 137:385-394.

27. Coulouarn C, Corlu A, Glaise D, Guenon I, Thorgeirsson SS and Clement B. Hepatocytestellate cell cross-talk in the liver engenders a permissive inflammatory microenvironment that drives progression in hepatocellular carcinoma. Cancer Res 2012; 72:2533-2542.

28. Barcena C, Stefanovic M, Tutusaus A, Joannas L, Menendez A, Garcia-Ruiz C, et al. Gas6/Axl pathway is activated in chronic liver disease and its targeting reduces fbrosis via hepatic stellate cell inactivation. J Hepatol 2015; 63:670-678.

29. Okabe H, Hayashi H, Nakagawa S, Imai K, Nitta $\mathrm{H}$, Arima $\mathrm{K}$, et al. Inducible factors for cancerassociated fbroblasts in liver cancer versus myofbroblasts in inflammatory liver disease. Histol Histopathol 2016; 31:141-148.

30. Trevisani F, D'Intino PE, Morselli-Labate AM, Mazzella G, Accogli E, Caraceni P, et al. Serum alphafetoprotein for diagnosis of hepatocellular carcinoma (HCC) in patients with chronic liver disease: influence of $\mathrm{HbsAg}$ and anti-HCV status. J Hepatol 2001; 34: 570-575.

31. Omran D, Esmat S, Sedrac H, Heba, ElBadry, Ahmad, et al. Can we use GP73 as a biomarker for the detection of hepatocellular carcinoma? Egyptian Liver Journal 2011; 1 (1): 43-46.

32. Xu MZ, Chan SW, Liu AM, Wong KF, Fan ST, Chen $\mathrm{J}$, et al. AXL receptor kinase is a mediator of YAP-dependent oncogenic functions in hepatocellular carcinoma. Oncogene 2011; 30(10):1229-1240

33. Pinato DJ, Brown MW, Trousil S, Aboagye EO, Beaumont J, Zhang $\mathrm{H}$, et al. Integrated analysis of multiple receptor tyrosine kinases identifies Axl as a therapeutic target and mediator of resistance to sorafenib in hepatocellular carcinoma. British Journal of Cancer 2019; 120: $512-521$ 